\title{
Genetics of Tracking of Body Mass Index from Birth to Late Middle Age: Evidence from Twin and Family Studies
}

\author{
Karri Silventoinen ${ }^{\mathrm{a}, \mathrm{b}}$ Jaakko Kaprio ${ }^{\mathrm{b}, \mathrm{c}}$

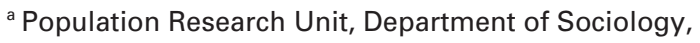 \\ ${ }^{\mathrm{b}}$ Department of Public Health, University of Helsinki, \\ ${ }^{\mathrm{c}}$ Department of Mental Health and Alcohol Research, National Public Health Institute, Helsinki, Finland
}

\section{Key Words}

Aging · BMI - Genetics · Weight gain · Obesity · Tracking

\begin{abstract}
Summary
The persistence of obesity from early childhood to late middle age is well known. We reviewed the results from existing genetic studies on tracking of BMI to discover how much genetic and environmental factors contribute to this tracking of obesity. In total, we found 5 genetic longitudinal studies on childhood obesity and 8 on obesity in adulthood. One was an adoption study, 3 were family studies, and 9 were twin studies. All were based on Caucasian populations, and one included genetic level information (the FTO gene). Strong genetic continuity in BMI was found from early childhood to onset of adulthood. Although new genetic factors started to affect $\mathrm{BMI}$ during the growth period, genetic correlations remained high. Evidence of the effect of common environment on the tracking of BMl during childhood was also found. The heritability estimates reported in twin studies ranged from 0.57 to 0.86 for the trend of BMI from early adulthood to late middle age. The three family studies gave lower estimates. Important unresolved questions are the genetics of BMI change in old age, the genetics of body composition change, the genetic architecture of tracking of obesity in ethnic groups other than Caucasians, and the interplay between genes and environment underlying the development and tracking of obesity.
\end{abstract}

\section{Introduction}

The most important risk factor for obesity at any phase of human life is being obese previously. This simple fact has important implications since it demonstrates how difficult it is to lose weight or even to prevent further weight gain. Understanding the processes underlying this tracking of obesity is crucial when searching for measures to prevent obesity. Childhood is a crucial stage of life when coping with this problem, since overweight children have a high risk of becoming obese adults [1,2]. However, weight loss or at least preventing weight gain is also possible in adulthood and can lead to important positive health consequences [3].

Twin and family studies have shown that genetic differences between individuals explain a major part of variation in BMI $[4,5]$. Thus genetic factors are likely to be important when explaining the persistence of BMI, although it is also possible that environmental factors contribute to it, especially in childhood. In this systematic review we set out to summarize evidence from existing genetic studies on factors contributing to the tracking of relative weight. Because the biological processes underlying weight gain are very different during the growth period and adulthood, studies on children and adults were treated separately.

\section{Search Strategy}

Our aim was to find all studies that had analyzed the genetic component behind the longitudinal change of BMI. Thus all eligible studies needed i) to include a measure of relative weight, ii) be longitudinal studies, and iii) have a genetically informative study design. We made a literature search in PubMed (www.pubmed.com) using the following formula: (body weight OR relative weight OR body mass index OR BMI) AND (change OR longitudinal) AND (heritability OR genetics OR twins OR twin study OR adoption study). Additionally, we limited our search to human studies published in English. We extended the search by reviewing the reference lists of the found articles and searching for articles that

\section{KARGER}

Fax +497614520714

Information@Karger.de

www.karger.com (c) 2009 S. Karger GmbH, Freiburg

Accessible online at:

www.karger.com/ofa 
had referred to these articles using the ISI Web of Knowledge (http://apps.isiknowledge.com). We found 5 studies on BMI in childhood [6-10] and 8 on BMI in adulthood [11-18]. Of the 13 studies finally included in this review, 1 involved adopted children [6], 3 focused on nuclear families [13-15], and the rest utilized information on monozygotic (MZ) and dizygotic (DZ) twins [7-12, 16-18].

\section{Methodological Aspects of Twin and Family Studies}

Regardless of the type of relatives included, family studies are based on an assumption that four sources of variation underlie the observed trait variation within a population. Genetic variation can be divided into i) additive genetic variation, which is the sum of the effects of all alleles affecting the trait, and ii) dominance genetic variation, which is caused by interaction between alleles in the same locus. An epistatic effect, i.e. interaction between alleles in different loci, is modeled as part of an additive or dominance genetic effect depending on how closely the loci are linked together. Environmental variation can be distinguished as iii) variation common to relatives, and iv) variation unique to each individual in the pedigree including measurement error.

A major advantage of family studies is that they allow assumptions to be made about the correlations of each of these four variance components between relatives. For example, MZ twins are genetically identical at the sequence level, and thus the correlation of both additive and dominance genetic effects is 1 . DZ twins and ordinary biological siblings share, on average, half of their segregating genes and thus the expected correlation is 0.5 for additive and 0.25 for dominance genetic effects. Further, MZ twins, DZ twins, ordinary biological siblings, and adopted siblings have a correlation of 1 for common environment and 0 for unique environment.

The simplest way to analyze the genetics of tracking of $\mathrm{BMI}$ is to decompose the variation in the change of BMI into genetic and environmental components based on the above assumptions. In the case of two measurements BMI change can be computed simply by subtraction, and in the case of several measurements by computing the slope of the regression line. This method has been utilized in some studies analyzing change of BMI or other obesity indicators in adulthood using regression-based methods $[12,14,15]$, comparisons of correlations between relatives [13], or linear structural equations [11]. However, most of the recent studies have utilized the more sophisticated longitudinal models presented in figure 1 that are based on linear structural equations. For the sake of simplicity, only additive genetic and specific environmental factors are shown, but common environment or dominance genetic factors can also be included in the models. In cases where only twins reared together are available, such as in all previous twin studies on BMI tracking, these two latter sources of variation cannot be modeled simultaneously.

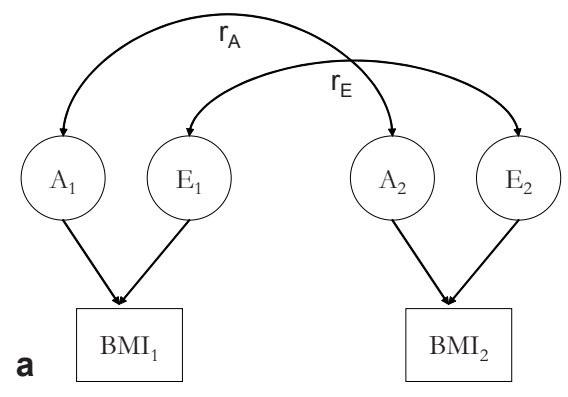

Fig. 1. Schematic representations of longitudinal models. a Cholesky decomposition including correlations of additive genetic $\left(\mathrm{r}_{\mathrm{A}}\right)$ and unique environmental factors $\left(\mathrm{r}_{\mathrm{E}}\right)$. b Simplex model including additive genetic and unique environmental innovations $\left(\zeta_{\mathrm{A} 1}-\zeta_{\mathrm{A} 3}\right.$ and $\zeta_{\mathrm{E} 1}-\zeta_{\mathrm{E} 3}$, respectively) and transmissions $\left(\beta_{\mathrm{A} 1}-\beta_{\mathrm{A} 2}\right.$ and $\beta_{\mathrm{E} 1}-\beta_{\mathrm{E} 2}$, respectively) and random errors $(\varepsilon)$. c Latent growth curve model including intercept $(\alpha)$ and slope $(\beta)$ of regression line and genetic $\left(\mathrm{r}_{\mathrm{A}}\right)$ and environmental correlations $\left(\mathrm{r}_{\mathrm{E}}\right)$ between them.

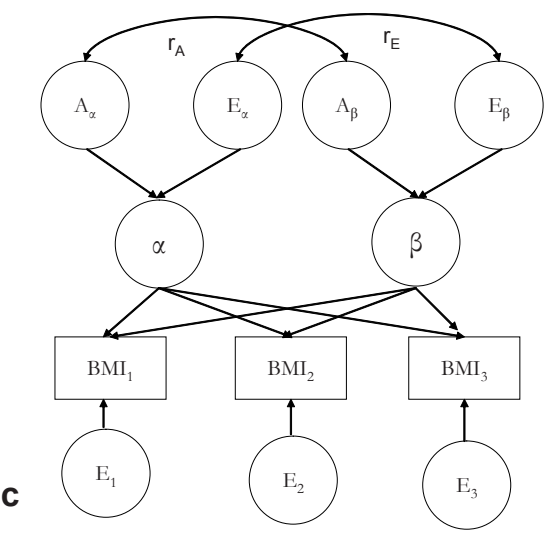

Cholesky decomposition is a way to compute genetic and environmental correlations. Figure 1a presents the Cholesky decomposition for BMI at two time points, although it is also possible to conduct it for multiple time points. Accordingly, the trait correlation between BMI at the two different ages is due to additive genetic correlation $\left(\mathrm{r}_{\mathrm{A}}\right)$ indicating the same or closely linked genes, and unique environmental correlation $\left(\mathrm{r}_{\mathrm{E}}\right)$ indicating the same or correlated environmental factors. Because the Cholesky decomposition makes no assumptions about the underlying genetic structure of the growth process but simply decomposes any variance and co-variance into new components, it is a suitable method if the nature of the growth process is not well known. However the Cholesky approach 
becomes unwieldy when the number of data points becomes large. Cholesky decomposition was applied in 3 studies of BMI change in childhood $[7,8,10]$ and in 1 study of BMI change in adulthood [16].

The Simplex model (fig. 1b) represents change in BMI as a dynamic process in which new genetic and environmental factors start to influence at each age. This new element of genetic or environmental variation is denoted as innovation parameters $\left(\zeta_{\mathrm{A} 1}-\zeta_{\mathrm{A} 3}\right.$ and $\zeta_{\mathrm{E} 1}-\zeta_{\mathrm{E} 3}$, respectively). Simultaneously, some or all of the factors having influence at the previous age may also still be important. This genetic or environmental persistence is denoted as transmission paths in the model $\left(\beta_{\mathrm{A} 1}-\beta_{\mathrm{A} 2}\right.$ and $\beta_{\mathrm{E} 1}-\beta_{\mathrm{E} 2}$, respectively). Further, an error variance term $(\varepsilon)$ is expected to affect each measure. Thus, the model permits analysis of whether new genetic or environmental variation starts to take effect during the period under study and whether part or all of the variation operating at previous ages continues to influence at later ages, too. At the phenotypic level, the Simplex model is based on the assumption that the size of correlations decreases simultaneously with increasing time between measurement points, and thus it is a suitable method for analyzing change of BMI in childhood. The Simplex model was used for this purpose in 2 studies $[6,9]$.

The third longitudinal model used in the included studies is the latent growth curve model (fig. 1c). This model allows estimation of the genetic and environmental variation affecting the intercept $(\alpha)$ and slope $(\beta)$ of the regression line. In addition, the model allows estimation of how these factors correlate with each other $\left(r_{\mathrm{A}}\right.$ for additive genetic correlation and $r_{E}$ for specific environmental correlation). Thus it is possible to analyze, for example, how much of the genes affecting baseline BMI (intercept) also affect the change of BMI (slope). Because the latent growth curve model in this formulation makes a strong assumption of linear change, it can be applied only to analyze change of BMI in adulthood, as was done in 2 studies [17, 18]. However, with more data points, quadratic or higher order slope terms can be added to describe departures from linearity. For example, a leveling off of rate of weight gain can be tested in this way.

\section{Tracking of BMI from Birth to Early Adulthood}

Table 1 summarizes information on the studies on BMI change in childhood. The earliest study was conducted in 1995 on US adopted children [6]. This study presented the Simplex model for the annual change of BMI from birth to 9 years of age. The transmission of

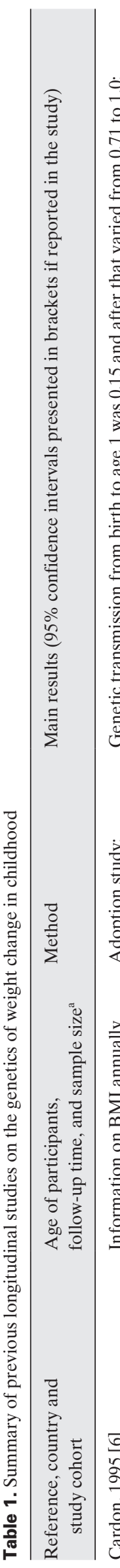

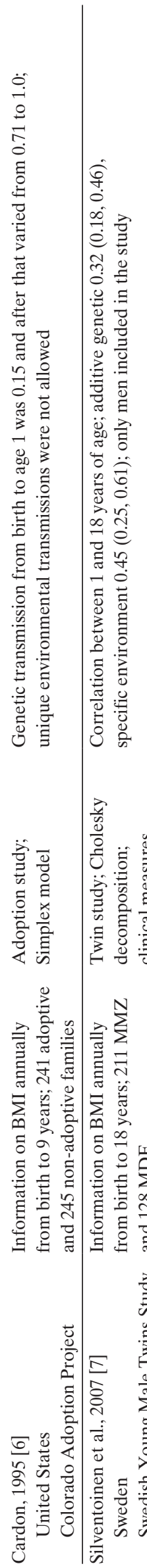
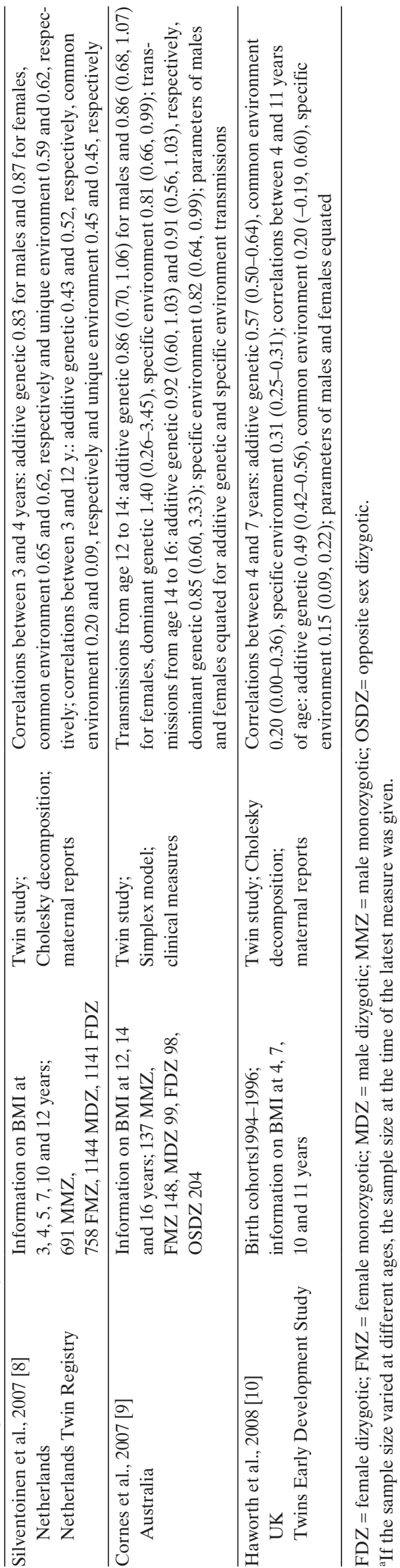
the additive genetic factors $\left(\beta_{\mathrm{A}}\right.$ parameters in fig. $\left.1 \mathrm{~b}\right)$ was 0.15 from birth to 1 year of age and after that was high, varying between 0.97 to 1.0 for most of the years. Unfortunately, it is not clear whether the model was correctly specified. It is particularly noteworthy that the model does not allow specific environmental transmissions ( $\beta_{\mathrm{E}}$ parameters in fig. $\left.1 \mathrm{~b}\right)$. This is an unrealistic assumption since BMI at any given age is a function of BMI at a previous age and BMI change; this trait continuity is modeled as part of specific environmental transmissions. It is also surprising that all transmission parameters were 1.0 at the highest; this may suggest that these components were unrealistically limited not to have higher values indicating increasing trait variation. However, even taking account of these limitations, this study provides evidence of strong genetic continuity in BMI from early to mid-childhood. Another study applying the Simplex model was based on Australian twin data and analyzed the change of BMI from 12 to 16 years of age [9]. Part of the genetic variation was found to be due to dominance genetic effects in this study. Moreover, genetic transmissions were high, i.e. 0.86 or more, showing that a large part of the genetic variation of BMI persisted over this age period.

The three other studies analyzing change of BMI in childhood applied the Cholesky decomposition to twin data. A study on Swedish boys analyzed BMI annually from birth to 18 years of age [7]. This study found that the genetic correlation ( $\mathrm{r}_{\mathrm{A}}$ in fig. 1a) between BMI at 1 and 18 years of age was already 0.32 and increased steadily after that. A Dutch study found that the genetic correlation between 3 and 12 years of age was 0.43 for boys and 0.52 for girls [8]; these correlations increased steadily when closer ages were analyzed. Very similar genetic correlations were found in a UK study on boys and girls [10]; in this study the genetic correlation between BMI at 4 and 11 years of age was 0.49 . This study also found that the FTO gene, a candidate gene found to explain approximately $1 \%$ of the variation of $\mathrm{BMI}$ in several adult populations [19], was associated with BMI from 7 years of age onwards. However, the FTO gene was able to explain only a small proportion of the genetic correlations of BMI. Both the Dutch and the UK study also found that there was a substantial common environmental factor contributing to the tracking of BMI. However, the common environmental correlations were lower than the additive genetic correlations in these studies.

To summarize, the studies examined show that there is strong genetic continuity in BMI from early childhood to the onset of adulthood. New genetic factors start to affect BMI during the growth period, but genetic correlations are still as high as 0.5 between BMI in early childhood and early adulthood; between closer ages the genetic correlations are substantially higher. In addition to the effect of genetic factors, common environment shows an important contribution to the tracking in BMI during childhood. This component was found to be statistically significant in only two studies $[8,10]$, but it is noteworthy that distinguishing common environmental effects from additive genetic effects needs more power than when estimating additive genetic effects only. Thus it is not surprising that the two studies which found this effect were the largest twin studies.

\section{Tracking of BMI from Early Adulthood to Late Middle Age}

Information on the studies of BMI change in adulthood is presented in table 2 . The first two studies analyzing genetics of BMI change were based on US twin data. A study on US male veterans found a substantial heritability of 0.70 for the BMI change in a 43-year follow-up starting at age 20 [11]. Similar substantial heritability was found in a study of females [12]. This study was based on only two measurement points, and the age variation was much larger at baseline. Depending on the used statistical method, this study gave a heritability estimate varying from 0.57 to 0.86 for the change of BMI. Even though the statistical models were insufficiently described in these two early twin studies, they provide evidence for a substantial genetic component behind weight change in both men and women.

Much lower estimates of heritability were found in the three studies analyzing nuclear families. In the two Canadian studies, the heritability of the change of BMI was 0.37 [13] and 0.23 [14]. These were also the only studies reporting heritability estimates for the change of waist circumference (0.39 [14]) and skinfold thickness (0.16 [13] and 0.12 [14]). It is worth noting that the age range at baseline in both studies was very wide and also included children. A third family study was based on the Framingham cohort in the USA [15]. This study found a moderate heritability of 0.24 for BMI change over 24 years, whereas for shorter follow-up periods the heritability estimates were low. The problem in all of these family studies, however, is that the models used for analyses are not sufficiently described, and thus it is not possible to evaluate their background assumptions.

The two studies using latent growth curve models (fig. 1c) were both partly based on the same Finnish twin cohort data. This cohort was also used in an earlier study on the genetics of weight change [20], but because this study used only two measurement points and is a subset of a later study [17], it is not mentioned in table 2. In a study following up men and women 20-46 years of age at baseline in 1971 to 1990 , substantial heritability estimates for rate of weight change of 0.58 for men and 0.64 for women were found. Interestingly, this study detected only a small genetic correlation $\left(\mathrm{r}_{\mathrm{A}}\right.$ in fig. 1c) between BMI level (intercept) and rate of BMI change (slope), suggesting that the set of genes affecting change of BMI is different from the genes affecting baseline BMI. A study based on a subsample of females of this cohort aged 37 50 years at baseline and followed up until 2004 found a similar 


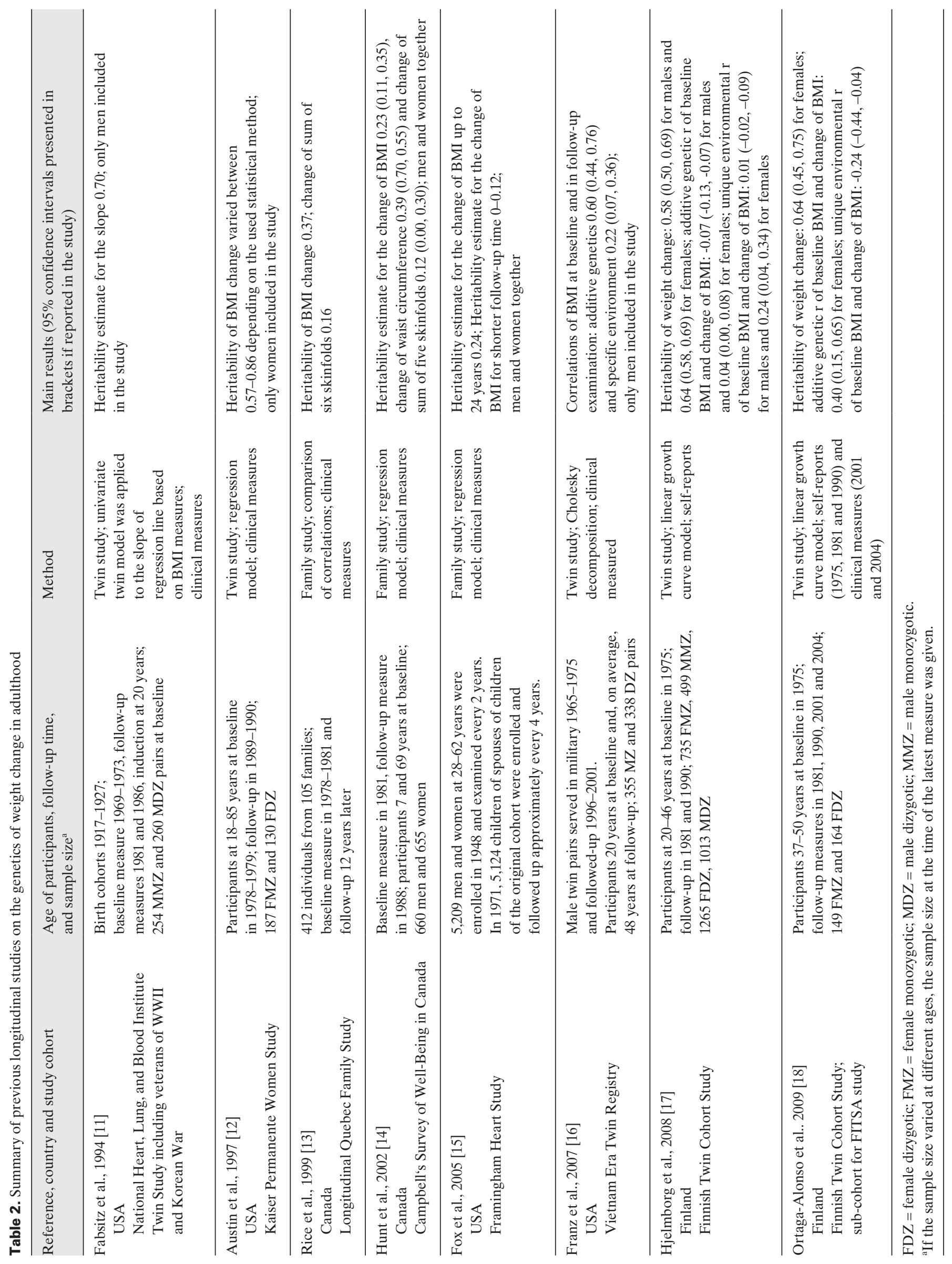


heritability of BMI rate of change, i.e. 0.64 [18]. In contrast to the study using the whole cohort, the genetic correlation between intercept and slope was substantial, i.e. 0.40. One possible explanation for these contrasting results is that this study also estimated a negative unique environmental correlation, which may have artificially strengthened the additive genetic correlation. Alternatively, it may be because it was a subset of the entire cohort, more restricted in age and with follow-up to older ages.

In conclusion, published twin studies have shown substantial heritability of BMI change in adulthood, varying from 0.57 to 0.86 using different measures of change, varying statistical models, and variable follow-up times. Family studies have yielded lower estimates. However, in these latter studies the statistical models were not described sufficiently, and therefore it is impossible to say whether these lower estimates are due to methodological limitations, different assumptions, or true sample differences. One issue which may have affected the lower genetic correlations is that the family studies were generally based on comparisons of relatives at very different ages, whereas twins are the same age. BMI also changes non-linearly with age, so simple adjustments by age will not fully adjust for age differences in BMI. Thus, if the genetic architecture is different in parents and their children, it may have decreased the estimates.

Intriguingly, the only studies analyzing the genetic correlation between baseline BMI and BMI change yielded somewhat different results even if they included partly the same subjects. However, the sample of female pairs from the Finnish Twin Cohort with more data points was much smaller in size, represented only selected birth cohorts, and covered later ages not included in the analysis of three data points of the entire Twin Cohort. Thus, the question of how much the genes affecting BMI also affect BMI change remains an open one. These preliminary results indicate that weight change needs to be considered as a separate phenotype from BMI in genome-wide searches for new genes.

\section{Further Prospects}

Taking into account the very large body of literature on obesity, it is somewhat surprising that there are only a few studies on the genetics of obesity tracking. A clear limitation of these studies is that they cover only the life span from birth to late middle age, with only one study offering any information on old age, i.e. over 60 years. Thus, more studies are needed on the genetics of weight change in elderly people. This would be an important issue to address since weight loss is found to be associated with higher mortality at old age [21]. It is likely that the genetics of weight change in elderly populations is very different from middle age populations, since weight starts to decrease in old age due to loss of muscle mass. Another limitation of existing studies is that all of them were conducted in
Caucasian populations. A recent twin study on the heritability of BMI and waist circumference in adolescents from eight countries found more genetic variation in Caucasian than in East-Asian populations [22]. Thus it would be important to analyze at which stage of the growth process this extra genetic variation emerges. Likewise, almost all of the existing studies have been based on BMI as the measure of obesity. Thus, genetically informative studies on changes in body composition over time are needed.

Knowledge of the specific genes affecting body fat and weight, such as FTO and $M C 4 R$, is accumulating. Once the known genes in combination account for a substantial fraction of the variance in BMI, their role in weight change needs to be assessed carefully. Both from the scientific and public health perspectives, it would also be important to analyze whether gene-environment interactions exist in the tracking of obesity. A recent study by Mustelin et al. [23], based on a Finnish twin cohort, revealed that among physically active young adults there was less genetic variation in BMI and waist circumference when compared to sedentary persons. This suggests that it may be possible to prevent expression of the genetic susceptibility to obesity by increased physical activity. It would be important to analyze whether high level of physical activity could also decrease genetic co-variation of obesity indicators between different ages. If this turns out to be true, it would emphasize the importance of physical activity in coping with the obesity epidemic.

\section{General Conclusions}

Previous family studies have shown strong genetic regulation of weight change from early childhood to late middle age. The underlying genes are the most important factor contributing to the tracking of obesity, but in childhood environmental factors are also important. Little is still known about candidate genes affecting this tracking. However, genetic and environmental factors are not likely to act independently, and further studies are needed to explore and reveal the interplay between nature and nurture underlying the development and tracking of obesity.

\section{Acknowledgements}

Support is acknowledged from the Academy of Finland Center of Excellence in Complex Disease Genetics and the DIOGENES projects which is the acronym for 'Diet, Obesity and Genes' supported by the European Community (Contract no. FP6-513946), www.diogenes-eu.org/. The authors thank Richard Burton, MA, for editing the text.

\section{Disclosure}

The authors declared no conflict of interest. 


\section{References}

1 Baird J, Fisher D, Lucas P, Kleijnen J, Roberts H, Law C: Being big or growing fast: systematic re view of size and growth in infancy and later obesity. BMJ 2005; 331:929.

2 Parsons TJ, Power C, Logan S, Summerbell CD Childhood predictors of adult obesity: a systematic review. Int J Obes Relat Metab Disord 1999;23 (suppl 8):S1-107.

3 Tuomilehto J, Lindström J, Silventoinen K: Obesity and prevention of type 2 diabetes; in Barnet AH, Kumar S (eds): Obesity and Diabetes. London, Wiley and Sons, 2004, pp 79-97.

4 Maes HH, Neale MC, Eaves LJ: Genetic and environmental factors in relative body weight and human adiposity. Behav Genet 1997;27:325-351.

5 Schousboe K, Willemsen G, Kyvik KO, Mortensen J, Boomsma DI, Cornes BK, et al: Sex differences in heritability of BMI: a comparative study of results from twin studies in eight countries. Twin Res 2003;6:409-421.

6 Cardon LR: Genetic influence on body mass index in early childhood; in Turner JR, Cardon LR (eds): Behavior Genetic Approaches in Behavioral Medicine. New York, Plenum Press, 1995, pp 133-143.

7 Silventoinen K, Pietiläinen KH, Tynelius $\mathrm{P}, \mathrm{S} \varnothing$ rensen TIA, Kaprio J, Rasmussen F: Genetic and environmental factors in relative weight from birth to age 18: the Swedish Young Male Twins Study. Int J Obes 2007;31:615-621.

8 Silventoinen K, Bartels M, Posthuma D, Estourgie-van Burk GF, Willemsen G, van Beijsterveldt TCEM, et al: Genetic regulation of growth in height and weight from three to 12 years of age: a longitudinal study of Dutch twin children. Twin Res 2007;10:354-363.
Cornes BK, Zhu G, Martin NG: Sex differences in genetic variation in weight: a longitudinal study of body mass index in adolescent twins. Behav Genet 2007;37:648-660.

10 Haworth CM, Carnell S, Meaburn EL, Davis OS, Plomin R, Wardle J: Increasing heritability of BMI and stronger associations with the FTO gene over childhood. Obesity 2008;16:2663-2668.

11 Fabsitz RR, Sholinsky P, Carmelli D: Genetic influences on adult weight gain and maximum body mass index in male twins. Am J Epidemiol 1994; 140:711-720.

12 Austin MA, Friedlander Y, Newman B, Edwards K, Mayer-Davis EJ, King MC: Genetic influences on changes in body mass index: a longitudinal analysis of women twins. Obes Res 1997;5:326-331.

13 Rice T, Perusse L, Bouchard C, Rao DC: Familial aggregation of body mass index and subcutaneous fat measures in the longitudinal Quebec family study. Genet Epidemiol 1999;16:316-334.

14 Hunt MS, Katzmarzyk PT, Perusse L, Rice T, Rao DC, Bouchard C: Familial resemblance of 7-year changes in body mass and adiposity. Obes Res 2002; 10:507-517.

15 Fox CS, Heard-Costa NL, Vasan RS, Murabito JM, D'Agostino RB Sr, Atwood LD, et al: Genomewide linkage analysis of weight change in the Framingham Heart Study. J Clin Endocrinol Metab 2005;90: 3197-3201.

16 Franz CE, Grant MD, Jacobson KC, Kremen WS, Eisen SA, Xian H, et al: Genetics of body mass stability and risk for chronic disease: a 28-year longitudinal study. Twin Res Hum Genet 2007;10:537545.
17 Hjelmborg JB, Fagnani C, Silventoinen K, McGue M, Korkeila M, Christensen K, et al: Genetic influences on growth traits of BMI: a longitudinal study of adult twins. Obesity 2008;16:847-852.

18 Ortaga-Alomso A, Sipilä S, Kujala UM, Kaprio J, Rantanen T: Genetic influences on change in BMI from middle to old age: a 29-year follow-up study of twin sisters. Behav Genet 2009;39:154-164.

19 Frayling TM, Timpson NJ, Weedon MN, Zeggini E, Freathy RM, Lindgren CM, et al: A common variant in the FTO gene is associated with body mass index and predisposes to childhood and adult obesity. Science 2007:316:889-894.

20 Korkeila M, Kaprio J, Rissanen A, Koskenvuo M: Consistency and change of body mass index and weight. A study on 5967 adult Finnish twin pairs. Int J Obes 1995;19:310-317.

21 Nguyen ND, Center JR, Eisman JA, Nguyen TV: Bone loss, weight loss, and weight fluctuation predict mortality risk in elderly men and women. $\mathrm{J}$ Bone Miner Res 2007;22:1147-1154.

22 Hur YM, Kaprio J, Iacono WG, Boomsma DI, McGue M, Silventoinen K, et al: Genetic influences on the difference in variability of height, weight and body mass index between Caucasian and East Asian adolescent twins. Int J Obes 2008;32:14551467.

23 Mustelin L, Silventoinen K, Pietiläinen K, Rissanen A, Kaprio J: Physical activity reduces the influence of genetic effects on BMI and waist circumference: a study in young adult twins. Int J Obes 2008;33:2936. 